CASE REPORT

\title{
Solitary fibrous tumour of the falciform ligament containing multiple foci of malignant transformation
}

\author{
A L Gidwani, F J Mullan, B Kenny
}

J Clin Pathol 2004;57:546-547. doi: 10.1136/jcp.2003.008821

Solitary fibrous tumour is an uncommon soft tissue tumour initially described in the pleural cavity. In the past decade, it has been described in various extrapleural sites, including the abdomen. Traditionally regarded as benign, cases of histologically or clinically malignant solitary fibrous tumours are rare. This report describes such a case, although the patient is still disease free five years after excisional surgery, without adjuvant treatment. The acute clinical presentation may be related to malignant transformation occurring in a long standing solitary fibrous tumour.

S olitary fibrous tumour is an uncommon soft tissue tumour initially described in the pleural cavity. In the past decade, it has been described in various extrapleural sites, including the abdomen. ${ }^{1}$ Traditionally regarded as benign, cases of histologically or clinically malignant solitary fibrous tumours are rare. We report such a case, in which acute clinical presentation may be related to malignant transformation occurring in a long standing solitary fibrous tumour.

\section{CASE REPORT}

A 50 year old man presented as an emergency with severe central upper abdominal pain in the absence of nausea, vomiting, or lower gastrointestinal symptoms. On examination, there was pronounced epigastric tenderness associated with an ill defined epigastric mass. He gave a history of previous ultrasonographic assessment of an epigastric mass eight years earlier, which had been thought to represent mesenteric duplication cysts.

On this occasion, enhanced computed tomography of the abdomen revealed a $16 \times 14 \times 9 \mathrm{~cm}$, mixed, attenuated, predominantly solid mass in the falciform ligament. It was not attached to an intraperitoneal organ, and appeared to be well encapsulated and well perfused on contrast enhancement. No evidence of ascites, lymphadenopathy, or metastasis was noted.

Laparotomy revealed a large mixed cystic/solid lesion within the falciform ligament, adherent to the posterior aspect of the rectus sheath. The vascular supply to the tumour appeared to arise from a pedicle in the base of the falciform ligament. The tumour was widely excised.

The gross surgical specimen was a large, rounded tumour, weight $1225 \mathrm{~g}$, measuring $16 \times 12 \times 10 \mathrm{~cm}$, with about two thirds of the surface being covered by peritoneum. Sectioning showed multiple rounded nodules of soft, focally necrotic tissue, ranging up to $7 \mathrm{~cm}$ in diameter, in a background of tough fibrotic tissue (fig 1).

Histologically, the background was fibrous tissue showing a typical patternless appearance, with variable (generally low) cellularity, and hyalinisation of a solitary fibrous tumour (fig $2 \mathrm{~A}-\mathrm{C}$ ). This gave way abruptly and multifocally

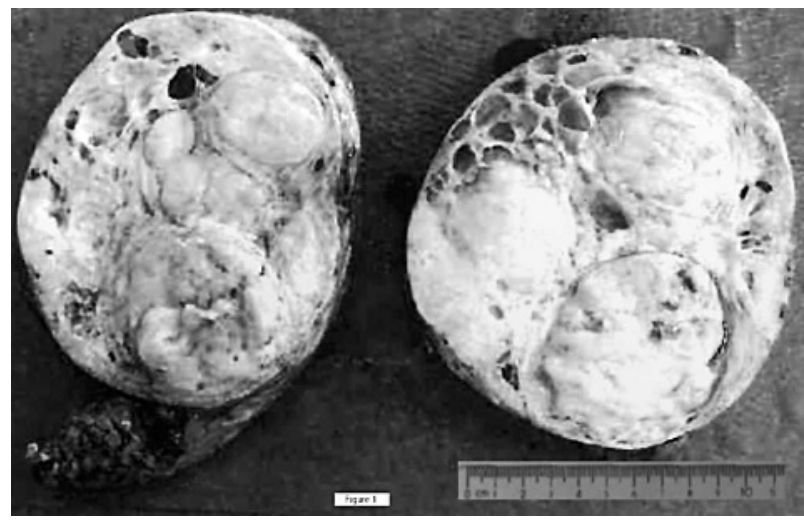

Figure 1 Gross pathology: cut surface of the tumour illustrating multinodularity.

to nodules of greatly increased cellularity, nuclear atypia, and focal necrosis-estimated at $25 \%$ of the cellular areas-with brisk mitotic activity (five mitosis/10 high power fields) (fig 2D). The surgical margins were found to be clear.

Both the hypocellular background and the cellular nodules were positive for CD34 and CD99, which is the characteristic immunophenotype of solitary fibrous tumours. It was clear that this lesion was a histologically malignant solitary fibrous tumour. Convalescence was uneventful and five years after surgery there is no clinical or radiological evidence of local recurrence or metastasis.

\section{DISCUSSION}

Tumours of the hepatic falciform ligament are rare. Leiomyosarcoma, lipoma, and endodermal sinus (yolk sac) tumours $^{2}$ have been reported to arise in the falciform ligament. Solitary fibrous tumours have been reported in various extrapleural sites, including the abdomen. We believe this to be the second reported case of a malignant solitary fibrous tumour arising from the falciform ligament; the first was reported in Japan in $1998 .^{3}$

One large review of 92 extrathoracic solitary fibrous tumours identified 10 that were clinically or histologically malignant. Recurrence and metastasis were not always predictable from the histology of the original tumour, and the authors argued that all cases of solitary fibrous tumour should be regarded as potentially malignant, ${ }^{4}$ although this is not the commonly held view. There is speculation as to whether or not malignant transformation can occur in benign solitary fibrous tumours. The history of a longstanding mass at the site, preceding an acute clinical presentation, and the morphology of the tumour, with overtly malignant nodules occurring in a background of benign looking solitary fibrous tumour, would support the view that such progresssion can occur in these lesions. Poor prognostic markers of solitary fibrous tumours include positive surgical margins, tumour 

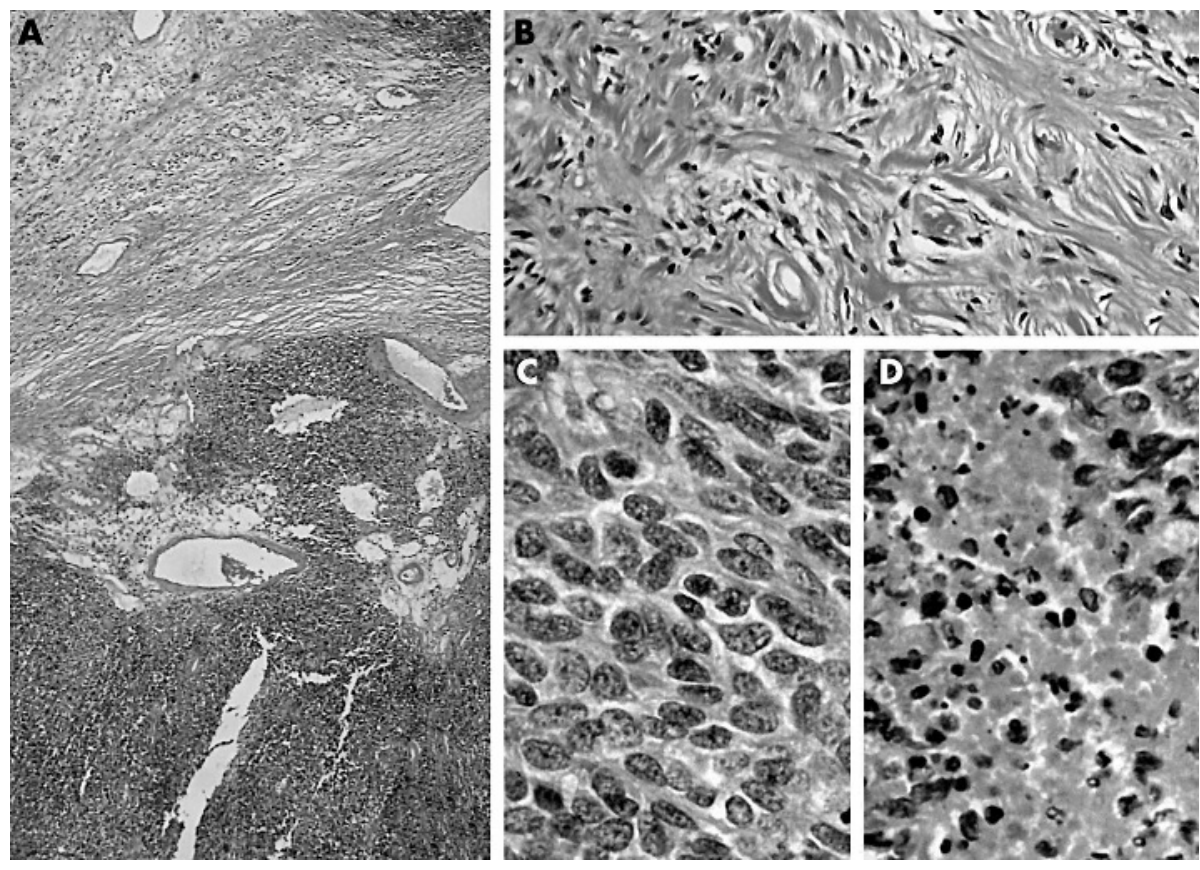

Figure 2 (A) Low power view of a cellular nodule and fibrous background. (B) The specimen had the classic appearance of a solitary fibrous tumour. (C) High power view of a cellular nodule. (D) Focal necrosis in a cellular nodule.

\section{Take home messages}

- We describe a rare case of malignant solitary fibrous tumour of the abdomen

- It is possible that the acute clinical presentation is related to malignant transformation occurring in a long standing solitary fibrous tumour

size more than $10 \mathrm{~cm}$, and mitotic activity of 10 mitosis/10 high power fields. ${ }^{5}$

"Recurrence and metastasis were not always predictable from the histology of the original tumour"

In this case, the patient is still disease free five years after excisional surgery, without adjuvant treatment. However, long term follow up will be necessary to determine whether the surgery has been curative. One could speculate that prognosis might be better in cases with malignant transformation where the malignant component has not spread beyond the edge of the benign counterpart.

\section{Authors' affiliations}

A L Gidwani, F J Mullan, Department of General Surgery, Causeway Hospitals Trust, 4 Newbridge Road, Coleraine, County Londonderry, BT52 1HS, UK

B Kenny, Department of Histopathology, Antrim Area Hospital, 45 Bush Road, Antrim BT41 2RL, UK

Correspondence to: A L Gidwani, Causeway Hospitals Trust, Number 2 Beeches Bungalows, 19 Maybrook Park, Mountsandel Road, Coleraine, County Londonderry, BT52 1SN, UK; gidwanianand@hotmail.com

Accepted for publication 12 November 2003

\section{REFERENCES}

1 Fukunaga $M$, Naganuma $\mathrm{H}$, Nikaido $\mathrm{T}$, et al. Extrapleural solitary fibrous tumour: a report of seven cases. Mod Pathol 1997;10:443-50.

2 Atkinson JB, Foster CE, Lally KP, et al. Primary endodermal sinus (yolk sac) tumour of the falciform ligament. J Paediatr Surg 1992;27:105-7.

3 Marubayashi S, Ohdan H, Asahara T, et al. Malignant mesothelioma originating in the hepatic falciform ligament: report of a case. Surg Today 1998;28:929-31.

4 Vallat-Decouvelaere AV, Dry SM, Fletcher CDM. Atypical and malignant solitary fibrous tumours in extra thoracic locations. Am J Surg Pathol 1998;22:1501-11.

5 Gold JS, Antonescu CR, Hajdu C, et al. Clinicopathological correlates of solitary fibrous tumours. Cancer 2002;94:1057-68. 